\title{
1 REV7/FANCV Binds to CHAMP1 and Promotes 2 Homologous Recombination Repair
}

Feng Li, Prabha Sarangi, Hanrong Feng, Lisa Moreau, Huy Nguyen, Connor Clairmont, Alan D. D’Andrea

1. Department of Radiation Oncology, Dana-Farber Cancer Institute, Boston, MA, 02215, USA

2. Center for DNA Damage and Repair, Dana-Farber Cancer Institute, Boston, MA, 02215, USA

Corresponding Author:

Alan D. D’Andrea, M.D.

Director: Center for DNA Damage and Repair

The Fuller-American Cancer Society Professor

Harvard Medical School

Chief, Division of Genomic Stability and DNA Repair

Department of Radiation Oncology

Dana-Farber Cancer Institute, HIM 243

450 Brookline Ave.

Boston, MA 02215

617-632-2080

FAX: 617-632-6069

Alan_Dandrea@dfci.harvard.edu

Running Title: REV7/CHAMP1 promotes Homologous Recombination

Keywords: REV7, TRIP13, CHAMP1, Homologous Recombination, Fanconi Anemia 
45 A critical determinant of DNA repair pathway choice is the HORMA protein REV7, a

46 small abundant adaptor which binds to various DNA repair proteins through its C-

47 terminal seatbelt domain. The REV7 seatbelt binds to the REV3 polymerase to form the

48 Polymerase $\zeta$ complex, a positive regulator of translesion synthesis (TLS) repair.

49 Alternatively, the REV7 seatbelt binds to SHLD3 in the Shieldin complex, a positive

50 regulator of NHEJ repair. Recent studies have identified another novel REV7 seatbelt-

51 binding protein, CHAMP1 (Chromosome Alignment-Maintaining Phosphoprotein, though

52 its role in DNA repair is unknown. Here, we show that the REV7-CHAMP1 complex

53 promotes homologous recombination (HR) repair by sequestering REV7 from the Shieldin

54 complex. CHAMP1 competes directly with the SHLD3 subunit of the Shieldin complex for

55 a limited pool of C-REV7, thereby inhibiting the REV7-mediated recruitment of the

56 SHLD2 and SHLD1 effector subunits to DNA double strand breaks. CHAMP1 thereby

57 channels DNA repair away from error-prone NHEJ and towards the competing error-free

58 HR pathway. Similarly, CHAMP1 competes with the REV3 component of the POL $\zeta$

59 complex, thereby reducing the level of mutagenic TLS repair. CHAMP1 interacts with

60 POGZ in a heterochromatin complex further promoting HR repair. Importantly, in

61 human tumors, CHAMP1 overexpression promotes HR, confers PARP inhibitor resistance,

62 and correlates with poor prognosis. Thus, by binding to either REV3, SHLD3, or

63 CHAMP1 through its seatbelt, the REV7 protein can promote either TLS repair, NHEJ

64 repair, or HR repair respectively. 


\section{INTRODUCTION}

REV7 (also known as MAD2L2, MAD2B, or FANCV), is a highly-conserved member of

69 the HORMA family of proteins, named for its three founding members: $\underline{\text { HOp}} 1$, a meiotic

70 chromosome axis factor, $\underline{\mathrm{REV}} 7$, and $\underline{\mathrm{MAD}} 2$, a spindle assembly checkpoint protein (Clairmont

71 and D'Andrea, 2021; de Krijger et al., 2021a). REV7 is an abundant cellular protein and is

72 unique among HORMA proteins, both in its large number of binding partners and in its

73 involvement in multiple distinct pathways. Germline biallelic mutations in the REV7 gene can

74 cause the inherited chromosome instability syndrome, Fanconi Anemia (Bluteau et al., 2016).

75 REV7 adopts the two classic closed and open seatbelt conformations of HORMA proteins, and

76 SHLD3 and REV3 are among its seatbelt dependent binding partners (Clairmont et al., 2020).

REV7 is an important determinant of DNA repair pathway choice (Clairmont and

D'Andrea, 2021). When closed REV7 (c-REV7) binds to SHLD3, this interaction promotes the assembly of the Shieldin complex (Findlay et al., 2018; Ghezraoui et al., 2018; Gupta et al.,

80 2018; Tomida et al., 2018). The Shieldin complex in turns blocks DSB end resection, promotes

81 reblunting of the resected DSBs, and promotes NHEJ (Dev et al., 2018; Gao et al., 2018; Mirman

82 et al., 2018; Noordermeer et al., 2018). When c-REV7 binds to REV3 in the POL $\zeta$ complex, the

83 interaction promotes error-prone Translesion Synthesis (TLS) Repair.

84 The AAA+ ATPase, TRIP13, along with its substrate adaptor $\mathrm{p} 31^{\text {comet }}$, can open REV7

85 and release SHLD3 or REV3 (Clairmont et al., 2020; Sarangi et al., 2020). Similarly, TRIP13

86 and $\mathrm{p} 31^{\text {comet }}$ are known to open other HORMA proteins, such as MAD2 (Brulotte et al., 2017;

87 Miniowitz-Shemtov et al., 2015; Ye et al., 2015). The mechanism by which REV7 is converted

88 from the inactive open conformation back to the active closed form is less well understood, and 
89 it may involve either the binding of another, unknown SBM-containing protein or a new post-

90 translational modification.

91 REV7 has at least one additional demonstrated seatbelt-binding partner, CHAMP1 (also

92 known as C13orf80, CAMP, or ZNF828). CHAMP1 is a little-known but highly conserved zinc

93 finger protein first identified as a REV7 interactor (Itoh et al., 2011). CHAMP1 localizes to

94 chromosomes, recruits REV7 to spindles, and plays a role in kinetochore-microtubule

95 interactions. Disruption of CHAMP1 leads to characteristic defects in chromosome alignment in

96 mitosis. Germline heterozygous mutations in CHAMP1 are associated with a rare syndromic

97 form of intellectual disability in humans (Isidor et al., 2016). Crystallographic analysis of the

98 REV7/CHAMP1 complex (Hara et al., 2017) revealed a strong similarity to the REV7/REV3 and

99 REV7/SHLD3 interaction surface (Hara et al., 2010). Despite the clear role of REV7 in DNA

100 repair pathway choice, little is known about the role of its interactor CHAMP1 in DNA repair.

101 Here, we demonstrate that the interaction of REV7 and CHAMP1 is required for DSB

102 end resection and error-free HR repair. CHAMP1 binds directly to the seatbelt domain of REV7

103 and thereby competes with the binding of SHLD3 and REV3. DNA damage and the ATM

104 kinase promote the closing of the REV7 seatbelt, resulting in the increased interaction of REV7

105 with all three binding partners. High cellular levels of CHAMP1 protein favor HR repair over

106 NHEJ and TLS and are often observed in human tumors with acquired HR proficiency.

107 Moreover, CHAMP1 is the active component of a large, multisubunit heterochromatin complex

108 containing HP1 $\alpha$, LEDGF, HDGFRP2, and POGZ previously shown to promote HR activity

109 (Baude et al., 2016; Clairmont et al., 2020; Daugaard et al., 2012; Nozawa et al., 2010;

110 Vermeulen et al., 2010). One function of this complex is to sequester REV7 away from other 
111 error-prone repair pathways, under specific cellular conditions and at specific regions of the 112 genome.

\section{RESULTS}

\section{REV7/CHAMP1 Complex promotes Homologous Recombination Repair}

117 In order to determine the possible involvement of CHAMP1 in DNA repair, we knocked

118 down CHAMP1 expression with siRNA in U2OS cells (Figure 1). Interestingly, CHAMP1

119 knockdown resulted in a reduction in HR activity, based on the decrease in GFP fluorescence

120 generated by the DR-GFP template versus the EJ5-GFP template (Pierce et al., 1999; Stark et al.,

121 2004) (Figure 1A, B and Figure S1A). Since an early step in HR repair is double strand break

122 (DSB) end resection (Symington, 2014), we used the SMART assay (Huertas and Cruz-Garcia,

123 2018) to quantify resection. Indeed, two siRNAs to CHAMP1 decreased DSB end-resection

124 (Figure 1C). Cells with an HR deficiency have a defect in RAD51 foci assembly and exhibit

125 sensitivity to PARP inhibitors (Bryant et al., 2005; Farmer et al., 2005). Accordingly, RPE-1

126 cells or U2OS cells with a CRISPR-Cas9-mediated knockout of CHAMP1 exhibited reduced

127 RAD51 foci (Figure 1D) and were sensitive to the PARP inhibitor, olaparib (Figure 1E, F and

128 Figure S1B-D). Previous studies have demonstrated that CHAMP1 interacts directly with

129 REV7 (Hara et al., 2017), a known regulator of DNA HR repair (Boersma et al., 2015; Xu et al.,

130 2015). To confirm and extend these findings, we showed that DNA damage with high dose UV

131 radiation activates the binding of CHAMP1 and REV7 and stimulates the colocalization of

132 REV7 and CHAMP1 in nuclear foci (Figure S1E-G). Moreover, CHAMP1 promotes the 
133 chromatin localization of REV7 (Figure S1H, I). Taken together, we reasoned that the DNA

134 damage inducible interaction of CHAMP1 and REV7 might be required for HR repair.

136 DNA damage activates REV7 seatbelt closure and partner protein binding

137 We next determined the mechanism by which DNA damage activates the closing of the 138 REV7 seatbelt (Figure 2). REV7 has a single, highly-conserved TQ site (T103) which is a 139 possible site of DNA damage-inducible, ATM-dependent phosphorylation (Figure S2A) 140 (Matsuoka et al., 2007). Interestingly, this TQ site aligns with a negatively-charged amino acid 141 (E105) in the primary sequence of another HORMA protein, MAD2. Moreover, in the closed 142 conformation of MAD2, an electrostatic interaction between E105 and the positively-charged 143 K192, likely contributes to the closing of the MAD2 seatbelt (Figure S2B). Similarly, REV7 144 has a K198 residue at the corresponding site. We therefore reasoned that a DNA-damage 145 inducible, ATM-dependent, phosphorylation of T103 of REV7 could account, at least in part, for 146 the DNA-damage inducible closing of REV7 and the binding of proteins with a SBM, such as 147 SHLD3, REV3, and CHAMP1. To test this hypothesis, we initially determined whether DNA 148 damage activates the phosphorylation of REV7 at T103, using an anti-p(S/T)Q antibody (Figure 149 S2C). Indeed, DNA damage activated the phosphorylation of REV7, and a point mutation of 150 REV7 (T103A) reduced this UV-activated phosphorylation of REV7 in vitro (Figure 2A) and its 151 chromatin recruitment (Figure 2B). Consistent with this, an ATM inhibitor reduced the DNA 152 damage-dependent phosphorylation of REV7, reduced the chromatin recruitment of REV7, and 153 decreased the assembly of REV7 foci (Figure 2C, D and Figure S2D, E). Similarly, UV 154 damage failed to activate the assembly of nuclear foci of the REV7-T103A mutant protein, 155 confirming that REV7 closing correlates with nuclear foci formation (Figure S2F). Unlike wild- 
type REV7, the REV7-T103A mutant protein failed to reduce RAD51 foci (Figure S2G, H) and

157 failed to restore PARP inhibitor sensitivity in REV7(-/-) cells (Figure S2I, J). Moreover,

158 knockdown of TRIP13 or p31 resulted in increased binding of CHAMP1 to REV7, while

159 overexpression of TRIP13 reduced this interaction, similarly to our previous findings with the

160 other seatbelt interactors SHLD3 and REV3 (Figure 2E, F). The REV7-T103A mutant

161 exhibited reduced binding to either SHLD3, CHAMP1, or REV3 (Figure 2G-I). Taken together,

162 DNA damage activates the ATM-dependent phosphorylation of T103 on REV7, thereby

163 promoting the closing of the REV7 seatbelt and the binding of SBM proteins, such as SHLD3

164 and CHAMP1. The TRIP13/p31 complex opens REV7 and releases these binding partners.

\section{CHAMP1 increases HR activity by competing with SHLD3 for binding to REV7}

167 REV7 is an abundant cellular protein, and it has several known binding partners (Noordermeer et

168 al., 2018). Some of these binding partners bind to the C-terminal seatbelt domain of REV7,

169 including SHLD3, REV3, and CHAMP1 (Clairmont and D'Andrea, 2021; de Krijger et al.,

170 2021a). We reasoned that these partners might compete for seatbelt binding under different

171 cellular conditions or cell cycle stages. The REV7 seatbelt binding protein, SHLD3, promotes

172 the assembly of the Shieldin Complex (Dev et al., 2018; Ghezraoui et al., 2018; Gupta et al.,

173 2018; Noordermeer et al., 2018), thereby blocking the resection of DSBs, recruiting the

174 CST/Pol $\alpha$ complex (Barazas et al., 2018; Mirman et al., 2018), and promoting blunt end ligation

175 via the NHEJ pathway. The TRIP13 ATPase, along with its binding partner $\mathrm{p} 31^{\text {comet }}$, opens the

176 seatbelt of REV7 and releases SHLD3 (Clairmont et al., 2020; Sarangi et al., 2020).

177 As CHAMP1 is much more abundant in cells than SHLD3 (Figure S3A), we determined 178 whether CHAMP1 regulates REV7 binding to SHLD3. In HEK293T cells, GFP-SHLD3 binds 
to REV7, and siRNA knockdown of CHAMP1 resulted in increased co-immunoprecipitation of

180 these proteins, demonstrating that CHAMP1 functions as a negative regulator of the Shieldin

181 complex (Figure 3A, B). The REV7 seatbelt also binds to REV3, and the REV7/REV3 (POLל)

182 complex promotes error-prone Translesion DNA Synthesis (TLS) and enhanced point

183 mutagenesis. We reasoned that CHAMP1 might also sequester REV7 from the REV7/REV3

184 complex and reduce error-prone TLS activity. To test this hypothesis, we generated and

185 expressed a GFP fusion protein containing the seatbelt binding domain of REV3. As predicted,

186 knocking out CHAMP1 resulted in an increased binding of the TLS polymerase subunit REV3 to

187 REV7 in U2OS cells (Figure 3C, D). Consistent with this result, CRISPR-Cas9-mediated

188 knockout of CHAMP1 in U2OS cells or RPE-1 cells resulted in increased REV7/REV3 activity,

189 as measured by MMC resistance and reduced MMC-induced chromosome radials (Figure 3E, F)

190 and Figure S3B-D). The TRIP13/p31 complex promotes the ATP-dependent opening of the

191 REV7/SHLD3 complex and releases SHLD3 (Clairmont et al., 2020). Taken together, these

192 results support a mechanism in which CHAMP1 promotes DSB end resection by sequestering

193 REV7 from SHLD3 and preventing the assembly of the Shieldin complex.

195 The REV7 binding activity of CHAMP1 is required for HR repair but not for proper

196 chromosome alignment

197 In its primary sequence, CHAMP1 has non-overlapping N-ZNF (C2H2-Zn finger

198 domains), SPE (PxxSPExxK motifs), WK (SPxxWKxxP motifs), FPE (FPExxK motifs), and C-

199 ZNF regions (Itoh et al., 2011) (Figure 4A). While the CHAMP1-WK region is required for

200 REV7 binding and recruitment of REV7 to spindles, the CHAMP1-FPE region appears to play

201 an independent role in chromosome alignment (Itoh et al., 2011). To confirm and extend these 
results, we generated two mutant forms of CHAMP1. According to the molecular structure of the REV7/CHAMP1 complex (Hara et al., 2017), the WKPAKPAPS - motif of CHAMP1, corresponding to the known consensus of a REV7 Seatbelt Binding Motif (SBM), interacts directly with the seatbelt domain of REV7 (Figure 4B), albeit with distinct amino acid residue interactions compared to the REV7/REV3 or the REV7/SHLD3 interactions. We therefore generated a mutant form of CHAMP1 which is predicted to disrupt this REV7 binding interaction (ie, the W334A/K335A double mutation, referred to as the CHAMP1-2A mutation). We also generated an in-frame deletion in CHAMP1 (del-FPE CHAMP1), previously shown to

210 be defective in the rescue of chromosome abnormalities in CHAMP1-/- cells (Itoh et al., 2011). As predicted, when expressed in RPE1 CHAMP1-/- cells, the CHAMP1-2A mutant

212 failed to bind to REV7, while the del-FPE CHAMP1 mutant was competent for REV7 binding

213 (Figure 4C). Indeed, the CHAMP1-2A mutant failed to correct the PARPi sensitivity of in

214 CHAMP1-/- cells, further confirming that REV7 binding and sequestration by CHAMP1 is

215 required for enhancement of HR activity (Figure 4D). The failure of CHAMP1-2A to restore

216 PARP inhibitor resistance and to increase MMC sensitivity was confirmed in the U2OS wild-

217 type or CHAMP1-/- cells (Figure S4A-E). Interestingly, complementation with the CHAMP1

218 del-FPE mutant yielded PARPi resistance, indicating that the FPE domain is not required for 219 enhancement of HR activity.

220 We next evaluated these two mutant proteins for their ability to correct chromosome

221 misalignment in CHAMP1-/- cells. Consistent with a previous report (Itoh et al., 2011),

222 CRISPR-knockout of CHAMP1 in RPE1 cells results in a severe defect in chromosome

223 alignment (Figure S4F, G). The CHAMP1 del-FPE mutant protein failed to complement the 224 chromosome misalignment and the G2/M accumulation of the CHAMP1-/- cells, but the WT 
225 CHAMP1 protein or the CHAMP1-2A mutant were functional in these assays (Figure 4E, F).

226 Taken together, the WK and FPE domains of CHAMP1 have independent, non-overlapping

227 functions. Moreover, REV7 binding to CHAMP1 is required for HR activity; however, the

228 CHAMP1-mediated recruitment of REV7 to the spindle is not required for the correction of

229 chromosome alignment.

\section{CHAMP1 regulates homologous recombination through REV7}

CHAMP1 therefore regulates HR activity through its ability to sequester REV7 from the

233 Shieldin complex. Thus, we expect that eliminating CHAMP1 in cells already lacking REV7

234 should not affect HR (Figure 5). HR activity can be scored by measuring the level of RAD51

235 foci or pRPA foci, both known to be increased in the setting of HR. As predicted siRNA

236 knockdown of CHAMP1 in wild-type RPE1 cells reduced the HR activity but failed to reduce

237 the HR activity in cells in which REV7 was already knocked out (Figure 5A-D). The REV7-/-

238 cells exhibited increased HR activity, as measured by PARP inhibitor resistance (Figure 5E),

239 regardless of their CHAMP1 expression level. Consistent with these results, knockdown of

240 CHAMP1 in a SHLD2-deficient cell line, HCC1937, also failed to cause Olaparib sensitivity

241 (Figure S5A).

242 To further validate this model, we next sought clinical evidence that CHAMP1

243 expression might affect cancer patient survival (Figure S5B, C). We reasoned that human

244 tumors with an underlying defect in an HR pathway might upregulate CHAMP1 as a

245 compensatory mechanism to tolerate their low HR and their replication stress. To test this

246 hypothesis, we correlated the level of CHAMP1 expression in ovarian tumors with patient

247 survival. For patients with tumors with low REV7 expression, the level of expression of 
CHAMP1 did not affect survival (Figure S5B). Thus, consistent with the cellular data, the

249 elevated HR activity in cells with low or absent REV7 expression was unaffected by CHAMP1

250 expression levels. Interestingly, for patients with tumors with high REV7 expression, the level

251 of CHAMP1 expression significantly affected patient survival (Figure S5C). The high

252 CHAMP1 expression correlated with a more aggressive tumor and poor patient prognosis,

253 perhaps resulting from the improved HR activity of these tumors. Taken together, the ability of

254 CHAMP1 to enhance HR is directly dependent on the presence of the REV7 protein.

256 CHAMP1 overexpression is common in tumors with underlying HR deficiency and

257 correlates with poor cancer patient prognosis

258 We next sought additional evidence that CHAMP1 upregulation correlates with PARP

259 inhibitor resistance. We used a panel of BRCA1-deficient cell lines with acquired PARPi-

260 resistance, collected through serial selection in increasing concentrations of PARPi (Figure 6A)

261 (Farkkila et al., 2021). These cells exhibited multiple independent mechanisms of PARPi

262 resistance, including downregulation of the Shieldin Complex or upregulation of ATR/CHK1

263 pathway activity (Farkkila et al., 2021). Interestingly, one of these PARPi-resistant clones

264 (NA5) exhibited high CHAMP1 protein expression compared to the parental PARPi-sensitive

265 cell line (Figure S6A). Knockdown of CHAMP1 in these cells restored PARPi sensitivity

266 (Figure 6B). In contrast, knockdown of CHAMP1 in another line (NA1), which has a lower

267 level of CHAMP1, did not restore PARPi sensitivity. Taken together, BRCAl-deficient cells can

268 acquire PARPi resistance, at least in part, by upregulating CHAMP1 expression.

269 Additional analysis of clinical databases revealed that, for patients with HR deficient

270 tumors containing a BRCA1 or BRCA2 mutation, a high level of CHAMP1 expression correlates 
271 with a worse prognosis (Figure 6C, D). This result further suggests that high CHAMP1

272 expression can partially correct the HR deficiency of these tumors, leading to a more aggressive

273 tumor phenotype. Tumors with BRCA2 mutations and presumably HR deficiency exhibited

274 higher baseline levels of CHAMP1 mRNA expression (Figure S6B). Consistent with these

275 observations, the level of CHAMP1 mRNA expression in cancer cell lines strongly correlates

276 with the level of CCNE1 mRNA expression (Figure 6E). Cells with a high degree of replication

277 stress resulting from CCNE1 amplification may therefore rely on CHAMP1-mediated HR for

278 their survival. Indeed, breast cancer cell lines with high expression of CCNE1 mRNA are more

279 dependent on CHAMP1 for their proliferation and survival (Figure 6F).

281 POGZ binds to CHAMP1 and cooperates in HR Repair

Recent studies have shown that CHAMP1 is a subunit of a large multisubunit complex of

283 HP1 $\alpha$ heterochromatin binding proteins. This complex includes HP1 $\alpha$, POGZ, LEDGF, and

284 HDGFRP2 (Baude et al., 2016; Clairmont et al., 2020; Daugaard et al., 2012; Nozawa et al.,

285 2010; Vermeulen et al., 2010). REV7 coimmunoprecipitates with multiple components of this

286 complex (Noordermeer et al., 2018), further suggesting a functional link with DNA repair

287 regulation. Interestingly, knockdown or knockout of many of the subunits of this complex, such

288 as HP1 $\alpha$ (Soria and Almouzni, 2013), LEDGF (Daugaard et al., 2012) or HDGFRP2 (Baude et

289 al., 2016), similar to the knockdown of CHAMP1, reduces DSB end resection and HR and

290 increases PARP inhibitor sensitivity (Olivieri et al., 2020). To confirm and extend these studies,

291 we next evaluated the POGZ subunit of this complex. Knockout of POGZ in RPE cells, like

292 knockout of CHAMP1, resulted in decreased HR repair, decreased DSB end resection, decreased

293 RAD51 foci, and increased PARP inhibitor sensitivity (Figure 7A-C and Figure S7A,B). 
294 Moreover, IR-induced DNA damage activated the co-immunopreciptation of POGZ and

295 CHAMP1 (Figure 7D). Knockdown of POGZ reduced the interaction of REV7 and CHAMP1

296 (Figure 7E), and POGZ binding to CHAMP1 was independent of REV7 seatbelt binding

297 (Figure S7C). Importantly, knockdown of POGZ in CHAMP(-/-) cells or knockdown CHAMP1

298 in POGZ(-/-) cells resulted in no additional impairment of HR repair or PARP inhibitor

299 sensitivity (Figure 7F, G), demonstrating that POGZ and CHAMP1 are epistatic in HR repair.

300 Finally, POGZ expression, like CHAMP1 expression, is increased in many human cancers,

301 consistent with its compensatory role in promoting HR repair (Figure S7D). Taken together,

302 these results demonstrate that a CHAMP1-containing multisubunit complex has a functional role

303 in sequestering REV7, preventing its association with SHLD3 in the Shieldin complex, and

304 promoting HR repair locally in heterochromatin.

305

306

307

\section{DISCUSSION}

Our results demonstrate that REV7, through its C-terminal seatbelt, can bind to three

309 different factors, SHLD3, REV3, and CHAMP1, to elicit distinct DNA repair outcomes. The

310 REV7-SHLD3 interaction mediates the assembly and accrual of the Shieldin complex at DSBs to

311 block DSB end resection and channel repair through NHEJ. The REV7-REV3 interaction

312 promotes the Pol $\zeta$ Translesion Synthesis (TLS) complex through its interaction with REV1, and

313 this complex bypasses bulky adducts of DNA during replication, thereby promoting mutagenesis.

314 Finally, the REV7-CHAMP1 complex, by sequestering REV7 from REV7-SHLD3 and REV7-

315 REV3 complexes, can promote error-free HR repair and function as a negative regulator of the

316 error-prone NHEJ and TLS repair pathways, respectively. Of the three binding proteins, 
317 CHAMP1 is the most abundant, resulting in a trend toward error-free DNA repair. The

318 mechanisms by which cells switch from one REV7 binding complex to another is largely

319 unknown.

320 Although CHAMP1 may have additional, independent roles in HR repair, its

321 sequestration of REV7 appears to be its primary mechanism in HR activity. Indeed, cells with a

322 knockout of REV7, versus cells with a double knockout of REV7 and CHAMP1, have equally

323 high levels of DSB end resection, RAD51 foci, and PARP inhibitor resistance. These results

324 argue for an epistatic relationship of REV7 and CHAMP1 in HR repair.

325 Previous studies indicate that TRIP13/p31 ${ }^{\text {comet }}$ complex opens REV7 and, like CHAMP1,

326 also reduces the Shieldin complex and promotes DSB end resection and HR repair (Clairmont et

327 al., 2020; de Krijger et al., 2021b). TRIP13 and CHAMP1 appear to be non-epistatic in HR

328 repair, however, since knockdown of TRIP13 in CHAMP1 (-/-) cells results in a further decline

329 in HR repair. Accordingly, a reduction in REV7/SHLD3 levels, by either TRIP13/p31 ${ }^{\text {comet }}$ or

330 CHAMP expression, appears to result from independent mechanisms for upregulating HR repair.

331 We have shown that DNA damage following ionizing radiation activates the ATM-

332 dependent closing of REV7 and the interaction of REV7 with either SHLD3, REV3, or

333 CHAMP1. How and when REV7 selectively chooses one binding partner versus another is

334 mostly unknown. It will be important to determine whether specific kinds of DNA damage will

335 preferentially activate a specific complex. For instance, IR may preferentially activate the

336 REV7/SHLD3 complex while replication fork perturbants, like hydroxyurea, may activate the

337 REV7/CHAMP1 complex. Also, the choice of a specific REV7 complex may be strongly

338 influenced by either the local organization of the genome, cell cycle cues, or cell type specificity.

339 The specific interaction of REV7 with SHLD3, REV3, or CHAMP1 may also be determined by 
340 the TRIP13/p31 ${ }^{\text {comet }}$ enzyme. While all three complexes are released by TRIP13/p31 ${ }^{\text {comet }}$, cells

341 may choose to selectively release one complex or another, depending on specific cellular

342 demands for NHEJ, TLS, or HR, respectively.

343 In addition to its role in the Shieldin complex and upregulation of NHEJ repair, REV7 is

344 also a critical determinant of Translesion DNA Synthesis (TLS repair). Indeed, one of the best

345 known roles of REV7 is its role as the small non-catalytic subunit of the Pol $\zeta$ complex. Pol $\zeta$ is

346 one of several human polymerases, specialized for synthesizing DNA across lesions in the

347 template strand, a process known as translesion synthesis (TLS). TLS is far more mutagenic than

348 normal replication (Goodman and Woodgate, 2013; Sale, 2013). In particular, DNA POL $\zeta$, in

349 conjunction with its partner, REV1, are responsible for the majority of spontaneous and damage

350 induced mutations during DNA replication (Gibbs et al., 2000; Jansen et al., 2005).

$351 \quad$ Although the mutagenic TLS and NHEJ pathways have historically been viewed as

352 independent processes, their coordinate regulation by REV7 calls for a reevaluation of this

353 relationship. Indeed, there are several important relationships between these processes aside from

354 REV7, and some of these relationships are functional or spatiotemporal. First, in both contexts,

355 the active REV7/REV3 or REV7/SHLD3 complexes promote the activity of rapid mutagenic

356 pathways, in contrast with the slower process of HR repair. Second, both TLS and NHEJ can

357 still play an important role during S-phase when the bulk of DNA repair is carried out HR. Lastly,

358 resection and the HR pathway are well-known to be utilized at stalled replication forks as well as

359 DSBs, raising the possibility that REV7/REV3 and REV7/SHLD3 could act on the same

360 substrate.

361 Finally, additional factors may influence the relative levels of REV7/SHLD3,

362 REV7/REV3, and REV7/CHAMP1 in the cell. First, REV7 may preferentially bind to 
363 CHAMP1 in heterochromatic regions of the genome, such as centromeres and telomeres,

364 resulting in higher local levels of HR repair. Second, CHAMP1 may also have a distinct

365 binding affinity for the REV7 seatbelt. Indeed, based on the corresponding crystal structures, the

366 molecular interactions of the REV7 seatbelt with either CHAMP1 or REV3 are distinct (Hara et

367 al., 2010; Hara et al., 2017) and likely to result in distinct binding affinities and off rates. Third,

368 some tumor cells with an underlying genetic deficiency in HR repair, such as a BRCA1 or

369 BRCA2 mutation, have higher level of expression of CHAMP1. Interestingly, this increase in

370 CHAMP1 may provide these cells with a compensatory increase in HR and a higher capacity for

371 tolerating replication stress. Finally, the specific interaction of REV7 with these various binding

372 partners may be highly regulated by post-translational modifications and under distinct cellular

373 conditions. Future studies are needed to further assess the spatial and temporal control of the

374 REV7 interaction with CHAMP1, SHLD3, and REV3.

\section{ACKNOWLEDGMENTS}

378 We thank all members of the D'Andrea laboratory for their helpful suggestions and

379 comments. This work was supported by grants from the US National Institutes of Health

380 (R37HL052725 and P01HL048546), the US Department of Defense (BM110181), the Breast

381 Cancer Research Foundation, the Fanconi Anemia Research Fund, the Ludwig Center at Harvard,

382 and the Smith Family Foundation (to A.D.D.) and the Claudia Adams Barr Program in

383 Innovative Basic Cancer Research (to F.L. and P.S). 
387 F.L., C.C., P.S., and A.D.D. conceived the study, analyzed the data, and wrote the manuscript.

388 H.F., L.M, and H.N. performed experiments and analyzed the data.

390 DISCLOSURES

391 A.D. D’Andrea is a consultant/advisory board member for Lilly Oncology, Merck-EMD Serono,

392 Cyteir Therapeutics, Third Rock Ventures, AstraZeneca, Ideaya Inc., and Cedilla Therapeutics

393 Inc. He is also a stockholder in Ideaya Inc., Cedilla Therapeutics Inc., and Cyteir Therapeutics,

394 and reports receiving commercial research grants from Lilly Oncology and Merck-EMD Serono.

395

396 


\section{FIGURE LEGENDS}

\section{Figure 1. CHAMP1 promotes homologous recombination}

399 A, Graph showing the percentage of GFP-positive cells after DR-GFP analysis. U2OS cells were

400 infected with I-SceI adenovirus and knocked down for BRCA1 or CHAMP1 using siRNA. N=3

401 biologically independent experiments. Error bars indicate standard errors, and p values were

402 calculated using two-tailed Student t-test, ***P<0.0001. B, Graph showing the percentage of

403 GFP-positive cells after EJ5-GFP analysis. U2OS cells were infected with I-SceI adenovirus and

404 knocked down for 53BP1 or CHAMP1 using siRNA. N=3 biologically independent experiments.

405 Error bars indicate standard errors, and p values were calculated using two-tailed Student t-test,

$406 * * * \mathrm{P}<0.0001, * * \mathrm{P}<0.001,{ }^{*} \mathrm{P}<0.05 . \mathrm{C}$, Quantification of resected ssDNA measured by SMART

407 assay in U2OS cells treated by siControl or siRNAs targeting CHAMP1 for 48hrs.

408 Approximately 50 fibers were counted per experiment. Error bars indicate standard errors, and $\mathrm{p}$

409 values were calculated using Student t-test, $* * * \mathrm{P}<0.0001$. D, (left) Representative images of

410 RAD51 foci formation in wild-type and two CHAMP1 knockout U2OS cell lines 6 hours after

411 5Gy IR treatment. (right) Quantification of >10 RAD51 foci. $n=3$ biologically independent

412 experiments. $* * * \mathrm{P}<0.001$. Statistical analysis was performed using two-tailed student's t-tests.

413 E, 5-day cytotoxicity analysis of wild type and two CHAMP1 knockout RPE1(p53-/-) cell lines

414 treated with various doses of Olaparib; $n=3$ independent experiments. Wild type versus

415 CHAMP1-KO\#1, ***P<0.0001; Wild type versus CHAMP1-KO\#2, ***P<0.0001; statistical

416 analysis was performed using two-way ANOVA. F, 3-day cytotoxicity analysis of wild type and

417 two CHAMP1 knockout U2OS cell lines treated with various doses of Olaparib. Cell viability

418 were detected by CellTiter-Glo (Promega)n=3 independent experiments. Wild type versus 
CHAMP1-KO\#1, ***P<0.0001; Wild type versus CHAMP1-KO\#2, ***P<0.0001; statistical

420 analysis was performed using two-way ANOVA.

Figure 2. DNA Damage Activates REV7 seatbelt closure and partner protein binding

A, 293T cells were transfected with FLAG-REV7-WT or FLAG-REV7-T103A, and following

424 treatment with/without UV $\left(20 \mathrm{~J} / \mathrm{m}^{2}\right)$ for 1 hour. The FLAG-immunoprecipitations were detected

425 by western blot using anti-Flag and anti-p-[S/T]Q antibodies. B, 293T cells were transfected with

426 FLAG-REV7-WT or FLAG-REV7-T103A, and following treatment with/without UV $\left(20 \mathrm{~J} / \mathrm{m}^{2}\right)$

427 as indicated. Western blot showing chromatin fraction of FLAG-REV7-WT and FLAG-REV7-

428 T103A. Histone H3 is used as control for chromatin isolation. C, Western blot showing

429 chromatin fraction of REV7 in U2OS treated with DMSO or ATM inhibitor, following IR

430 treatment as indicated. D, Schematic of our proposed model showing that the conformational

431 state is regulated by TRIP1-p31 complex and ATM. ATM phosphorylates REV7 at T103 site

432 and promotes the closed form of REV7. The closed REV7 interacts with CHAMP1, SHLD3 and

433 REV3. E, Western blot showing GFP-immunoprecipitation of GFP-CHAMP1 in wild-type (WT),

434 TRIP13 $3^{--}$and p31 ${ }^{--}$U2OS cells, and the co-immunoprecipitation of endogenous REV7. F,

435 Western blot showing GFP-immunoprecipitation of GFP-CHAMP1 in U2OS-vector control (VC)

436 and TRIP13 overexpressed U2OS cells, and the co-immunoprecipitation of endogenous TRIP13

437 and REV7. G, 293T cells were co-transfected with GFP-SHLD3 and Flag-REV7 or Flag-REV7-

438 T103A. Western blot showing GFP-immunoprecipitation of GFP-SHLD3, and the co-

439 immunoprecipitation of Flag-REV7 and Flag-REV7-T103A. H, Western blot showing Flag-

440 immunoprecipitation of Flag-REV7 wild type and Flag-REV7-T103A mutant, and the co-

441 immunoprecipitation of endogenous CHAMP1. I, 293T cells were co-transfected with GFP- 
442 tagged fragment of REV3 containing the REV7-binding domain (R3BD) and Flag-REV7 or

443 Flag-REV7-T103A. Western blot showing Flag-immunoprecipitation of Flag-REV7 and Flag-

444 REV7-T103A, and the co-immunoprecipitation of GFP-R3BD. All of the immunoblots are

445 representative of at least two independent experiments.

447 Figure 3. CHAMP1 competes with SHLD3 and REV3 for binding to REV7

448 A, Western blot showing GFP-immunoprecipitation of GFP-SHLD3 in 293T cells, treated with

449 or without siCHAMP1, and the co-immunoprecipitation of endogenous CHAMP1 and REV7. B,

450 Quantification of the relative SHLD3-REV7 binding activity from three independent

451 immunoprecipitation western blot shown in A. C. Western blot showing GFP-

452 immunoprecipitation of GFP-tagged fragment of REV3 containing the REV7 binding domain

453 (R3BD) in U2OS wild type and U2OS ${ }^{\text {CHAMP1-/- }}$ cells, and the co-immunoprecipitation of

454 endogenous CHAMP1 and REV7. D, Quantification of the relative REV3-REV7 binding activity

455 from three independent immunoprecipitation western blot shown in C. E, A 14 days clonogenic

456 assay of U2OS wild type and two CHAMP1-KO U2OS cell lines, treated with various doses of

457 MMC; $\mathrm{n}=3$ independent experiments. ${ }^{*} \mathrm{P}<0.05$. Statistical analysis was performed using two-way

458 ANOVA. F, A 14 days clonogenic assay of RPE1 ${ }^{\text {p53-/- }}$ and two RPE1 $1^{\text {p53-/-CHAMP1-/- }}$ cell lines,

459 treated with various doses of $\mathrm{MMC} ; \mathrm{n}=3$ independent experiments. $* \mathrm{P}<0.05$. Statistical analysis

460 was performed using two-way ANOVA.

462 Figure 4. The REV7 binding region of CHAMP1 is required for the HR function but not

463 for correction of chromosome misalignment 
464 A, (Top) Schematic of CHAMP1 protein showing its various domains and REV7 binding region.

465 (Bottom) Schematic of CHAMP1-Full Length (FL) and two mutants (2A and $\triangle F P E)$. SBM,

466 REV7 seatbelt binding motif. B, Structure of the REV7-CHAMP1 complex. REV7 is shown in

467 cyan and blue (seatbelt domain), and the CHAMP1 fragment (residues 331-343) is shown in red.

468 C, Western blot showing GFP-immunoprecipitation of GFP-Empty Vector, GFP-CHAMP1

469 wild-type, GFP-CHAMP1-2A mutant or GFP-CHAMP1- $\triangle F P E$, and the co-immunoprecipitation

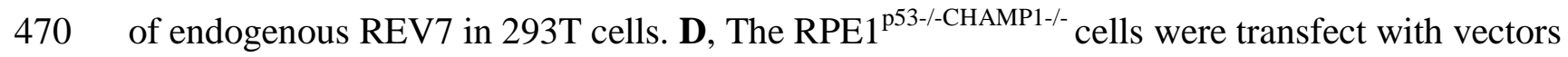

471 containing GFP-tagged CHAMP1-Full Length (FL) or mutants cDNA for 48 hours. GFP positive

472 cells were sorted by Flow Cytometry. A 14 days clonogenic assay of indicated cell lines treated

473 with various doses of Olaparib; $n=3$ independent experiments, $* * P<0.001$. Statistical analysis

474 was performed using two-way ANOVA. E, Summary of chromosome misalignment in indicated

475 cell lines from D. F, Same cell lines from D were fixed with $70 \%$ ethanol and stained with

476 propidium iodide. Quantitative analysis of indicated cells in G2/M were shown.

478 Figure 5. CHAMP1 regulates HR through REV7

479 A, Representative images of RAD51 foci formation in wild-type and $R E V 7^{-/}$U2OS cells treated 480 with siRNA negative control (siNC) and siCHAMP1, and 6 hours after 5Gy IR treatment. B,

481 Quantification of RAD51 in A. More than 10 RAD51 foci were counted. $n=3$ biologically 482 independent experiments, $* * * \mathrm{P}<0.0001$. Statistical analysis was performed using two-tailed 483 student's t-tests. C, Representative images of p-RPA32(S33) foci formation in wild-type and $484 R E V 7^{-/}$U2OS cells treated with siRNA negative control (siNC) and siCHAMP1, and 6 hours 485 after 5 Gy IR treatment. D, Quantification of p-RPA32(S33) foci in C. More than 10 RAD51 foci 486 were counted. $\mathrm{n}=3$ biologically independent experiments, $* * * \mathrm{P}<0.0001$. Statistical analysis was 
performed using two-tailed student's t-tests. E, (left) A 14-days clonogenic survival of wild-type and $\mathrm{REV7}^{-/-} \mathrm{U} 2 \mathrm{OS}$ cells treated with various doses of Olaparib after siControl or siCHAMP1 treatment. $\mathrm{n}=3$ independent experiments, $* * \mathrm{P}<0.001, * * * \mathrm{P}<0.0001$. Statistical analysis was

490 performed using two-way ANOVA. (right) A cartoon shows that CHAMP1 inhibits REV7 to 491 promote HR.

Fig 6. CAMP overexpression is common in tumors with underlying HR deficiency and correlates with poor cancer patient prognosis

495 A, Schematic of PARPi-resistant RPE1 $1^{\text {p53-/-BRCA1-/- }}$ cells generation. RPE1 ${ }^{\text {p53-/-BRCA1-/- }}$ cells (B40)

496 were treated with increasing concentrations of the PARPis niraparib/Olaparib over 3 months, and

497 then isolated by single-cell clones from the niraparib- and Olaparib-resistant pools. B, A 14-days 498 clonogenic survival of RPE1 ${ }^{\mathrm{p} 53-/-}, \mathrm{RPE}^{\mathrm{p} 53-/-\mathrm{BRCA} 1-/-}$ and niraparib/Olaparib-resistant RPE1 ${ }^{\mathrm{p} 53-/-}$

499 BRCA1-/ cell clones treated with various doses of Olaparib after siControl or siCHAMP1 treatment. $500 \mathrm{n}=3$ independent experiments. $* * * \mathrm{P}<0.0001$. Statistical analysis was performed using two-way

501 ANOVA. C-D, Kaplan-Meier curves depicting overall survival of patients from TCGA with

502 CHAMP1 expression and wildtype BRCA1 and BRCA2 (C), and mutated BRCA1 or BRCA2

503 (D). This analysis combines tumors from these TCGA studies: BLCA (blader), BRCA (breast),

504 LUAD (lung), LUSC (lung squamous), and SKCM (skin).

505 E, CHAMP1 expression positively correlates with Cyclin E expression. F, Breast cancer cells 506 with high expression of CCNE1 are more dependent on CHAMP1 for survival. 
510 A, Graph showing the percentage of GFP-positive cells after DR-GFP analysis. U2OS cells were

511 infected with I-SceI adenovirus and knocked down for BRCA1 or POGZ using siRNA. N=3

512 biologically independent experiments. Error bars indicate standard errors, and p values were

513 calculated using two-tailed Student t-test, $* * * \mathrm{P}<0.0001$. B, Quantification of resected ssDNA

514 measured by SMART assay in U2OS cells treated by siControl or siRNAs targeting CHAMP1

515 for $48 \mathrm{hrs}$. Approximately 50 fibers were counted per experiment. Error bars indicate standard

516 errors, and $\mathrm{p}$ values were calculated using Student t-test, $* * * \mathrm{P}<0.0001$. C, Quantification of $>10$

517 RAD51 foci in wild-type and two CHAMP1 knockout U2OS cell lines 6 hours after 5Gy IR

518 treatment. $\mathrm{n}=3$ biologically independent experiments. $* * * \mathrm{P}<0.001$. Statistical analysis was

519 performed using two-tailed student's t-tests. D, Western blot showing GFP-immunoprecipitation

520 of GFP-REV7 in HEK293T cells treated with or without irradiation (5Gy), and the co-

521 immunoprecipitation of endogenous CHAMP1 and POGZ. E, Western blot showing GFP-

522 immunoprecipitation of GFP-CHAMP1 in HEK293T cells treated with or without siPOGZ, and

523 the co-immunoprecipitation of endogenous CHAMP1 and POGZ. F, 3-day cytotoxicity analysis

524 of RPE1 $1^{\mathrm{p} 53-/-}$ and RPE1 $1^{\mathrm{p} 53-/-\mathrm{CHAMP1-/}}$ cells treated with various doses of Olaparib after 48 hours

525 siControl or siPOGZ treatment. Cell viability were detected by CellTiter-Glo (Promega), $\mathrm{n}=3$

526 independent experiments. Statistical analysis was performed using two-way ANOVA. G, 3-day

527 cytotoxicity analysis of RPE1 ${ }^{\text {p53-/- }}$ and RPE1 $1^{\text {p53-/-POGZ-/- }}$ cells treated with various doses of

528 Olaparib after 48 hours siControl or siCHAMP1 treatment. Cell viability were detected by

529 CellTiter-Glo (Promega), n=3 independent experiments. Statistical analysis was performed using

530 two-way ANOVA. All of the immunoblots are representative of at least two independent

531 experiments. 


\section{Materials and Methods}

\section{Cell culture and transfections}

536 Human U2OS, RPE1-hTERT, HCC1937 and HEK293T cells were cultured in DMEM/F12 +

537 Glutamax (Invitrogen) supplemented with 10\% FBS (Sigma) and 1\% penicillin-streptomycin

538 (Invitrogen). DNA transfections and siRNA knockdowns were carried out using Lipofectamine

539 LTX (Invitrogen) and RNAiMax (Invitrogen) respectively according to the manufacturer's

540 protocols. The individual siRNAs used are: AllStar negative siControl (1027281); siCHAMP1 \#4

541 (SI00973084); siCHAMP1 \#8 (SI04282159); siBRCA1 (SI00930510); si53BP1 (SI01456539)

542 were purchased from Qiagen. ON-target Human siPOGZ (L-006953-01-0005) were purchased

543 from Horizon Discovery.

\section{$544 \quad$ Antibodies and chemicals}

545 Antibodies used in this study were: Abnova H00283489-B01P (C13orf8/CHAMP1, IB, IF),

546 Abcam ab180579 (Mad2L2/REV7, IB, IF), Bethyl Laboratories A302-509A (POGZ, IB, IF),

547 Abcam ab128171 (TRIP13, IB), Cell Signaling 3873 (alpha-Tubulin, IF), Cell Signaling 2187

548 (Phospho-CENP-A, IF), Cell Signaling 3638 (H3, IB), Cell Signaling 2956 (GFP, IB), Cell

549 Signaling 3700 (Actin, IB), Abcam ab70369 (phospho-Kap1-S824, IB), Cell Signaling 6966

550 (Phospho-[S/T]Q, IB), Fisher Scientific NB100544 (RPA2-P-Ser33, IF), Santa Cruz sc-8349

551 (RAD51, IF), Millipore-Sigma F3165 (GAPDH, IB) and Millipore-Sigma F3165 (Flag, IB, IF).

552 Mitomycin C (MMC) was purchased from Sigma and Olaparib was purchased from Selleckchem.

\section{Generation of knockout cell lines with CRISPR-Cas9}

554 CHAMP1 and POGZ guide RNA sequences were cloned into the pSpCas9 BB-2A-GFP (PX458)

555 vector (GenScript). U2OS and RPE1 ${ }^{\text {p53-/- }}$ cells were transfected with Cas9-gRNA plasmids.

556 After 48 hours GFP positive cells were selected using a BD FACSAria II cell sorter. Single cells 
557 from GFP positive pool were cultured for three to four weeks and colonies were screened for

558 knockouts by western blotting using the anti-p31 ${ }^{\text {comet }}$ antibody (Millipore-Sigma). The guide

559 RNA sequences targeting CHAMP1 in this study were: \#1 TCGTAAACCATCAGCACGTT and

560 \#2 CCAGAGATCCGTAGTCCAGC. The guide RNA sequences targeting POGZ in this study

561 were: \#1 CAGTTTGTTAAGCCGACAGT and \#2 TCTGCTGATCGAGTTCTACG.

\section{GFP-based DNA Repair Assays}

563 For DR- and EJ5-GFP reporter assays, U2OS cells carrying the respective GFP expression

564 cassette were transfected with the indicated siRNAs. 24 hours after transfection, cells were

565 infected with or without I-SceI lentivirus. After 48 hours, cells were harvested and detected by

566 flow cytometry. The data was analyzed using the FlowJo software.

\section{Cellular fractionation and immunoblot analysis}

568 Cells were lysed with NP40 buffer (1\% NP40, $300 \mathrm{mM} \mathrm{NaCl}, 0.1 \mathrm{mM}$ EDTA, $50 \mathrm{mM}$ Tris (pH

569 7.5)) supplemented with phosphatase and protease inhibitor cocktail (Roche). Cell lysates were

570 resolved by NuPAGE 4-12\% Bis-Tris gels (Invitrogen), and transferred onto nitrocellulose

571 membranes. Membranes were blocked with 5\% BSA in TBST and were sequentially incubated

572 with primary and secondary antibodies and detected using chemiluminescence or fluorescence

573 (LI-COR Biosciences). For chromatin extraction, chromatin-bound extracts were got using

574 subcellular protein fractionation kit (Thermo). The band intensities were measured by ImageJ.

\section{Immunoprecipitation}

576 After transfection for 48h, 293T or U2OS cells were then harvested and lysed in NETN lysis

577 buffer with proteinase \& phosphatase inhibitor cocktail (Thermo, 1:100) for 30 minutes on ice.

578 They were then incubated with antibody-bead conjugate (Anti-FLAG® M2 Magnetic Beads,

579 Millipore \& Sigma or GFP-Trap_A, Chromotek) overnight at $4{ }^{\circ} \mathrm{C}$. Beads were washed four 
580 times with NETN buffer and immunoprecipitates were eluted by boiling. Western blots were

581 performed to detect the immunoprecipitates. The band intensities were measured by ImageJ.

582 Drug sensitivity assays

583 Cells were transfected with plasmid or siRNA $24 \mathrm{~h}$ before being plated for colony formation or

584 CellTiter-Glo assays. To assay clonogenic survival, cells were seeded at 500-1000 cells/well in

585 6-well plates in triplicates. Drugs at the shown doses were added after 12 hours and cells were

586 permitted to grow for 14 days. Colony formation was scored by fixing and staining with $0.5 \%$

587 (w/v) crystal violet in $20 \%$ methanol. For short term CellTiter-Glo survival assays, cells were

588 plated in 96-well plates at 800-1000 cells/well, and treated with drugs at the indicated

589 concentrations after 12 hours. Three days later, cellular viability was measured using CellTiter-

590 Glo (Promega). Survival at each drug concentration was calculated as a percentage normalized to

591 the corresponding untreated control, for both assays.

\section{Immunofluorescence assays}

593 Cells were plated on glass coverslips in 12-well plates. They were then either left untreated or

594 treated at $20 \mathrm{~J} / \mathrm{m}^{2} \mathrm{UV}$ or $5 \mathrm{~Gy}$ IR. After 1 or 6 hours, they were harvested by pre-extraction with

$595 \quad 0.5 \%$ Triton X-100 for $5 \mathrm{~min}$, followed $4 \%$ paraformaldehyde fixation for $10 \mathrm{~min}$ at $4{ }^{\circ} \mathrm{C}$. After

596 three PBS washes, blocking was performed with 3\% BSA in PBS for 1 hour at room temperature,

597 followed by sequential primary and secondary antibody incubations overnight at $4{ }^{\circ} \mathrm{C}$ and 1 hour

598 at room temperature respectively. The coverslips were mounted with DAPI (Vector

599 Laboratories) and captured using a Zeiss AX10 fluorescence microscope and Zen software, and

600 foci were scored. At least 100 cells were counted for each sample.

\section{SMART assay}


602 The SMART DNA fiber assay procedure was performed largely as described previously

603 (Clairmont et al., 2020). In brief, cells were treated with BrdU (sigma) for 24h, and then exposed

604 to X-ray irradiation to induce DSB formation. Cells were collected $6 \mathrm{~h}$ after irradiation, and

605 embedded in low melting point agarose plugs before lysis with proteinase $\mathrm{K}$ overnight at $50^{\circ} \mathrm{C}$.

606 The plugs were then washed with TE buffer and digested with agarase (NEB). The sample

607 solution was spread onto silanized coverslips using the FiberComb machine (Genomic Vision).

608 Combed coverslips were blocking with 3\% BSA for 30min, and then incubated with anti-BrdU

609 antibody (rat, abcam) overnight at $4^{\circ} \mathrm{C}$. After incubation with secondary Alexa-555-labelled goat

610 anti-rat antibodies, the coverslips were washed and mounted with Vectashield mounting medium

611 (Vector laboratories). Images were captured by Zeiss AX10 flurorescence microscope. At least

612100 fibers were counted per condition. The fiber lengths were measured using imageJ and

613 graphed.

\section{Chromosomal aberration analysis}

$615 \mathrm{RPE}^{\mathrm{p53-/-}}$ and RPE1 $1^{\mathrm{p53-/-CHAMP1-/-}}$ cells were incubated with or without $20 \mathrm{ng} / \mathrm{ml} \mathrm{MMC} \mathrm{for} 48$

616 hours. Cells were treated with $100 \mathrm{ng} / \mathrm{ml}$ of colcemid for 2 hours, followed by a hypotonic

617 solution $(0.075 \mathrm{M} \mathrm{KCl})$ for $20 \mathrm{~min}$ and fixed with 3:1 methanol/acetic acid. After staining with

618 Wright's stain, 50 metaphase spreads were counted for aberrations. The relative number of

619 chromosomal dicentrics and radials was calculated relative to control cells as indicated.

\section{TCGA data acquisition and analysis}

621 The survival analyses of the Cancer Genome Atlas (TCGA) patients were performed using the

622 clinical and RNASeq expression and genomic alteration data of TCGA Pan-Cancer study for 32

623 cancer types downloaded from the cBioPortal for Cancer Genomics (https://www.cbioportal.org;

624 retrieved March 2020). For the survival analysis with mRNA expression of CHAMP1 
625 (CHAMP1) and REV7 (MAD2L2), for each cancer type, samples were grouped into the low-

626 and high-mRNA expressing groups for CHAMP1 and REV7 based on the expression z-scores of

627 either zero, or less than -0.5 and greater than 0.5 . These expression z-scores were computed

628 relative to the diploid samples. Survival analysis was then performed in $\mathrm{R}$ for each cancer type

629 to determine whether there was a difference in the overall survival between the two groups,

630 separately for CHAMP1 and REV7, and for REV7 in each of the two CHAMP1 groups. Kaplan-

631 Meier curves were created, and the log-rank test was used to test for a difference in overall

632 survival using the survival package in $\mathrm{R}$. The $\mathrm{p}$ values were calculated from the chi-square

633 distribution. The survminer R package was used to estimate median survivals, and to plot the

634 Kaplan-Meier curves. Additionally, Cox proportional hazards regression was performed to

635 estimate the hazard ratio between the low- and the high-mRNA groups for each cancer type.

636 The survival analyses of TCGA patients with CHAMP1 mRNA expression and mutation

637 status of BRCA1 and BRCA2 were performed as follows. A tumor was considered mutated for a

638 gene if it had variants with classifications that were damaging or other non-conserving. The

639 analyses were first performed for each cancer type and independently with mutation status of

640 BRCA1 and BRCA2. Then the cancer types that showed a trend from the results with either gene

641 were selected for the combined analysis. The combined survival analysis with CHAMP1

642 expression and mutation status of BRCA1 and BRCA2 was performed with tumors being

643 considered mutated if they had a mutated status for either BRCA1 or BRCA2. Also, the Cox

644 proportional hazards regression was performed with accounting for the differences between

645 cancer types and between tumor stages.

646 Cancer cell lines' data acquisition and analysis 
The association analyses between cyclin E (CCNE1) expression and CHAMP1 dependency

648 and CHAMP1 expression in cancer cell lines were performed using the expression data from the

649 Cancer Cell Line Encyclopedia (CCLE) project (Ghandi et al., 2019) and the dependency data

650 from the Broad Institute Cancer Dependency Map (DepMap; CRISPR DepMap Public 19Q4

651 dataset) (Meyers et al., 2017). Both datasets were downloaded from the DepMap Portal

652 (https://depmap.org/portal/). The RNASeq expression counts were normalized by the TMM

653 (weighted trimmed mean of M-values) method using the edgeR package (Robinson et al., 2010)

654 and transformed into $\log 2$-counts per million $(\log 2-\mathrm{CPM})$ values. For each cancer lineage, the

655 low- and high-cyclin E mRNA expressing groups were determined using the median log2-CPM.

656 The significance of the difference in the CHAMP1 dependency between the low- and high-cyclin

657 E mRNA groups were assessed by the Wilcoxon rank sum test using the ggpubr R package. The

658 correlation between CHAMP1 mRNA expression and cyclin E mRNA expression were

659 performed by the simple linear regression on the log2-CPM values using the ggpmisc R package.

660 The plots were generated using the ggplot2 package in $\mathrm{R}$. 


\section{REFERENCES}

664

Barazas, M., Annunziato, S., Pettitt, S.J., de Krijger, I., Ghezraoui, H., Roobol, S.J., Lutz, C., Frankum, J., Song, F.F., Brough, R., et al. (2018). The CST Complex Mediates End Protection at Double-Strand Breaks and Promotes PARP Inhibitor Sensitivity in BRCA1-Deficient Cells. Cell Rep 23, 2107-2118.

Baude, A., Aaes, T.L., Zhai, B., Al-Nakouzi, N., Oo, H.Z., Daugaard, M., Rohde, M., and Jaattela, M. (2016). Hepatoma-derived growth factor-related protein 2 promotes DNA repair by homologous recombination. Nucleic Acids Res 44, 2214-2226.

Bluteau, D., Masliah-Planchon, J., Clairmont, C., Rousseau, A., Ceccaldi, R., Dubois d'Enghien, C., Bluteau, O., Cuccuini, W., Gachet, S., Peffault de Latour, R., et al. (2016). Biallelic inactivation of REV7 is associated with Fanconi anemia. J Clin Invest 126, 3580-3584.

Boersma, V., Moatti, N., Segura-Bayona, S., Peuscher, M.H., van der Torre, J., Wevers, B.A., Orthwein, A., Durocher, D., and Jacobs, J.J.L. (2015). MAD2L2 controls DNA repair at telomeres and DNA breaks by inhibiting 5' end resection. Nature 521, 537-540.

Brulotte, M.L., Jeong, B.C., Li, F., Li, B., Yu, E.B., Wu, Q., Brautigam, C.A., Yu, H., and Luo, X. (2017). Mechanistic insight into TRIP13-catalyzed Mad2 structural transition and spindle checkpoint silencing. Nat Commun 8, 1956.

Bryant, H.E., Schultz, N., Thomas, H.D., Parker, K.M., Flower, D., Lopez, E., Kyle, S., Meuth, M., Curtin, N.J., and Helleday, T. (2005). Specific killing of BRCA2-deficient tumours with inhibitors of poly(ADP-ribose) polymerase. Nature 434, 913-917.

Clairmont, C.S., and D'Andrea, A.D. (2021). REV7 directs DNA repair pathway choice. Trends Cell Biol.

Clairmont, C.S., Sarangi, P., Ponnienselvan, K., Galli, L.D., Csete, I., Moreau, L., Adelmant, G., Chowdhury, D., Marto, J.A., and D'Andrea, A.D. (2020). TRIP13 regulates DNA repair pathway choice through REV7 conformational change. Nat Cell Biol 22, 87-96.

Daugaard, M., Baude, A., Fugger, K., Povlsen, L.K., Beck, H., Sorensen, C.S., Petersen, N.H., Sorensen, P.H., Lukas, C., Bartek, J., et al. (2012). LEDGF (p75) promotes DNA-end resection and homologous recombination. Nat Struct Mol Biol 19, 803-810.

de Krijger, I., Boersma, V., and Jacobs, J.J.L. (2021a). REV7: Jack of many trades. Trends Cell Biol.

de Krijger, I., Fohr, B., Perez, S.H., Vincendeau, E., Serrat, J., Thouin, A.M., Susvirkar, V., Lescale, C., Paniagua, I., Hoekman, L., et al. (2021b). MAD2L2 dimerization and TRIP13 control shieldin activity in DNA repair. Nat Commun 12, 5421.

Dev, H., Chiang, T.W., Lescale, C., de Krijger, I., Martin, A.G., Pilger, D., Coates, J., Sczaniecka-Clift, M., Wei, W., Ostermaier, M., et al. (2018). Shieldin complex promotes DNA end-joining and counters homologous recombination in BRCA1-null cells. Nat Cell Biol 20, 954-965.

Farkkila, A., Rodriguez, A., Oikkonen, J., Gulhan, D.C., Nguyen, H., Dominguez, J., Ramos, S., Mills, C.E., Perez-Villatoro, F., Lazaro, J.B., et al. (2021). Heterogeneity and Clonal Evolution of Acquired PARP Inhibitor Resistance in TP53- and BRCA1-Deficient Cells. Cancer Res 81, 2774-2787.

Farmer, H., McCabe, N., Lord, C.J., Tutt, A.N., Johnson, D.A., Richardson, T.B., Santarosa, M., Dillon, K.J., Hickson, I., Knights, C., et al. (2005). Targeting the DNA repair defect in BRCA mutant cells as a therapeutic strategy. Nature 434, 917-921. 
Findlay, S., Heath, J., Luo, V.M., Malina, A., Morin, T., Coulombe, Y., Djerir, B., Li, Z., Samiei, A., Simo-Cheyou, E., et al. (2018). SHLD2/FAM35A co-operates with REV7 to coordinate DNA double-strand break repair pathway choice. EMBO J 37.

711 Gao, S., Feng, S., Ning, S., Liu, J., Zhao, H., Xu, Y., Shang, J., Li, K., Li, Q., Guo, R., et al. 712 (2018). An OB-fold complex controls the repair pathways for DNA double-strand breaks. Nat Commun 9, 3925.

Ghandi, M., Huang, F.W., Jane-Valbuena, J., Kryukov, G.V., Lo, C.C., McDonald, E.R., 3rd, Barretina, J., Gelfand, E.T., Bielski, C.M., Li, H., et al. (2019). Next-generation characterization of the Cancer Cell Line Encyclopedia. Nature 569, 503-508. Ghezraoui, H., Oliveira, C., Becker, J.R., Bilham, K., Moralli, D., Anzilotti, C., Fischer, R., Deobagkar-Lele, M., Sanchiz-Calvo, M., Fueyo-Marcos, E., et al. (2018). 53BP1 cooperation with the REV7-shieldin complex underpins DNA structure-specific NHEJ. Nature 560, 122-127. Gibbs, P.E., Wang, X.D., Li, Z., McManus, T.P., McGregor, W.G., Lawrence, C.W., and Maher, V.M. (2000). The function of the human homolog of Saccharomyces cerevisiae REV1 is required for mutagenesis induced by UV light. Proc Natl Acad Sci U S A 97, 4186-4191.

Goodman, M.F., and Woodgate, R. (2013). Translesion DNA polymerases. Cold Spring Harb Perspect Biol 5, a010363.

Gupta, R., Somyajit, K., Narita, T., Maskey, E., Stanlie, A., Kremer, M., Typas, D., Lammers, M., Mailand, N., Nussenzweig, A., et al. (2018). DNA Repair Network Analysis Reveals Shieldin as a Key Regulator of NHEJ and PARP Inhibitor Sensitivity. Cell 173, 972-988 e923.

Hara, K., Hashimoto, H., Murakumo, Y., Kobayashi, S., Kogame, T., Unzai, S., Akashi, S., Takeda, S., Shimizu, T., and Sato, M. (2010). Crystal structure of human REV7 in complex with a human REV3 fragment and structural implication of the interaction between DNA polymerase zeta and REV1. J Biol Chem 285, 12299-12307.

Hara, K., Taharazako, S., Ikeda, M., Fujita, H., Mikami, Y., Kikuchi, S., Hishiki, A., Yokoyama, H., Ishikawa, Y., Kanno, S.I., et al. (2017). Dynamic feature of mitotic arrest deficient 2-like protein 2 (MAD2L2) and structural basis for its interaction with chromosome alignmentmaintaining phosphoprotein (CAMP). J Biol Chem 292, 17658-17667.

Huertas, P., and Cruz-Garcia, A. (2018). Single Molecule Analysis of Resection Tracks. Methods Mol Biol 1672, 147-154.

Isidor, B., Kury, S., Rosenfeld, J.A., Besnard, T., Schmitt, S., Joss, S., Davies, S.J., Lebel, R.R., Henderson, A., Schaaf, C.P., et al. (2016). De Novo Truncating Mutations in the KinetochoreMicrotubules Attachment Gene CHAMP1 Cause Syndromic Intellectual Disability. Hum Mutat 37, 354-358.

Itoh, G., Kanno, S., Uchida, K.S., Chiba, S., Sugino, S., Watanabe, K., Mizuno, K., Yasui, A., Hirota, T., and Tanaka, K. (2011). CAMP (C13orf8, ZNF828) is a novel regulator of kinetochore-microtubule attachment. EMBO J 30, 130-144.

Jansen, J.G., Tsaalbi-Shtylik, A., Langerak, P., Calleja, F., Meijers, C.M., Jacobs, H., and de Wind, N. (2005). The BRCT domain of mammalian Rev1 is involved in regulating DNA translesion synthesis. Nucleic Acids Res 33, 356-365.

748 Matsuoka, S., Ballif, B.A., Smogorzewska, A., McDonald, E.R., 3rd, Hurov, K.E., Luo, J., 749 Bakalarski, C.E., Zhao, Z., Solimini, N., Lerenthal, Y., et al. (2007). ATM and ATR substrate analysis reveals extensive protein networks responsive to DNA damage. Science 316, 1160-1166. Meyers, R.M., Bryan, J.G., McFarland, J.M., Weir, B.A., Sizemore, A.E., Xu, H., Dharia, N.V., Montgomery, P.G., Cowley, G.S., Pantel, S., et al. (2017). Computational correction of copy 
number effect improves specificity of CRISPR-Cas9 essentiality screens in cancer cells. Nat Genet 49, 1779-1784.

Miniowitz-Shemtov, S., Eytan, E., Kaisari, S., Sitry-Shevah, D., and Hershko, A. (2015). Mode of interaction of TRIP13 AAA-ATPase with the Mad2-binding protein p31comet and with mitotic checkpoint complexes. Proc Natl Acad Sci U S A 112, 11536-11540. Zimmermann, M., Durocher, D., and de Lange, T. (2018). 53BP1-RIF1-shieldin counteracts DSB resection through CST- and Polalpha-dependent fill-in. Nature 560, 112-116. Noordermeer, S.M., Adam, S., Setiaputra, D., Barazas, M., Pettitt, S.J., Ling, A.K., Olivieri, M., Alvarez-Quilon, A., Moatti, N., Zimmermann, M., et al. (2018). The shieldin complex mediates 53BP1-dependent DNA repair. Nature 560, 117-121. Obuse, C. (2010). Human POGZ modulates dissociation of HP1alpha from mitotic chromosome arms through Aurora B activation. Nat Cell Biol 12, 719-727. Damage in Human Cells. Cell 182, 481-496 e421.

Pierce, A.J., Johnson, R.D., Thompson, L.H., and Jasin, M. (1999). XRCC3 promotes homologydirected repair of DNA damage in mammalian cells. Genes Dev 13, 2633-2638.

Robinson, M.D., McCarthy, D.J., and Smyth, G.K. (2010). edgeR: a Bioconductor package for differential expression analysis of digital gene expression data. Bioinformatics 26, 139-140.

Sale, J.E. (2013). Translesion DNA synthesis and mutagenesis in eukaryotes. Cold Spring Harb Perspect Biol 5, a012708.

Sarangi, P., Clairmont, C.S., Galli, L.D., Moreau, L.A., and D'Andrea, A.D. (2020). p31(comet) promotes homologous recombination by inactivating REV7 through the TRIP13 ATPase. Proc Natl Acad Sci U S A 117, 26795-26803.

779 Soria, G., and Almouzni, G. (2013). Differential contribution of HP1 proteins to DNA end resection and homology-directed repair. Cell Cycle 12, 422-429.

Stark, J.M., Pierce, A.J., Oh, J., Pastink, A., and Jasin, M. (2004). Genetic steps of mammalian homologous repair with distinct mutagenic consequences. Mol Cell Biol 24, 9305-9316.

Symington, L.S. (2014). End resection at double-strand breaks: mechanism and regulation. Cold Spring Harb Perspect Biol 6.

Tomida, J., Takata, K.I., Bhetawal, S., Person, M.D., Chao, H.P., Tang, D.G., and Wood, R.D. (2018). FAM35A associates with REV7 and modulates DNA damage responses of normal and BRCA1-defective cells. EMBO J 37.

Vermeulen, M., Eberl, H.C., Matarese, F., Marks, H., Denissov, S., Butter, F., Lee, K.K., Olsen, J.V., Hyman, A.A., Stunnenberg, H.G., et al. (2010). Quantitative interaction proteomics and genome-wide profiling of epigenetic histone marks and their readers. Cell 142, 967-980.

Xu, G., Chapman, J.R., Brandsma, I., Yuan, J., Mistrik, M., Bouwman, P., Bartkova, J., Gogola, E., Warmerdam, D., Barazas, M., et al. (2015). REV7 counteracts DNA double-strand break resection and affects PARP inhibition. Nature 521, 541-544.

794 Ye, Q., Rosenberg, S.C., Moeller, A., Speir, J.A., Su, T.Y., and Corbett, K.D. (2015). TRIP13 is

795

796 a protein-remodeling AAA+ ATPase that catalyzes MAD2 conformation switching. Elife 4. 

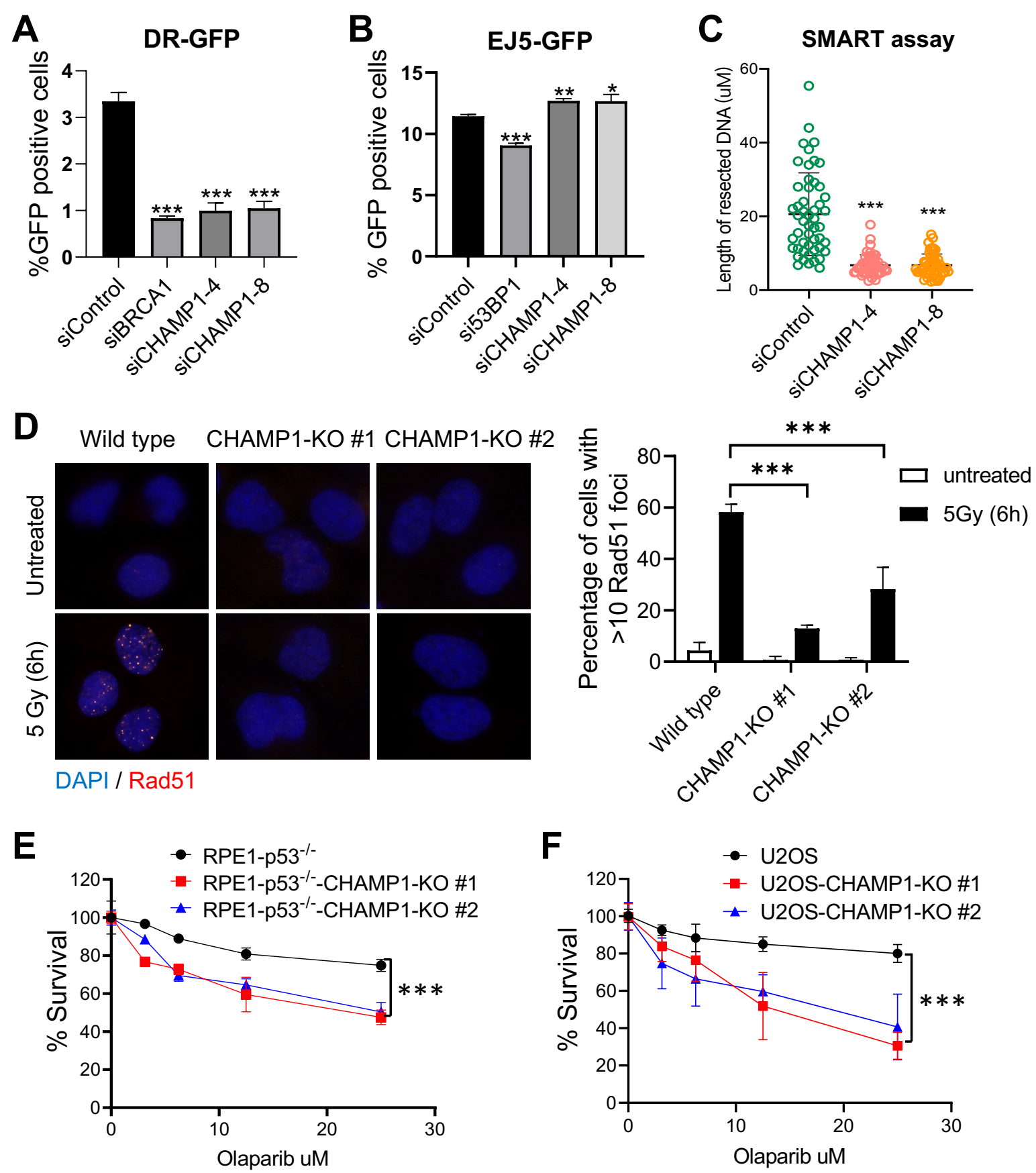
Fig 2. DNA Damage Activates REV7 seatbelt closure and partner protein binding

A

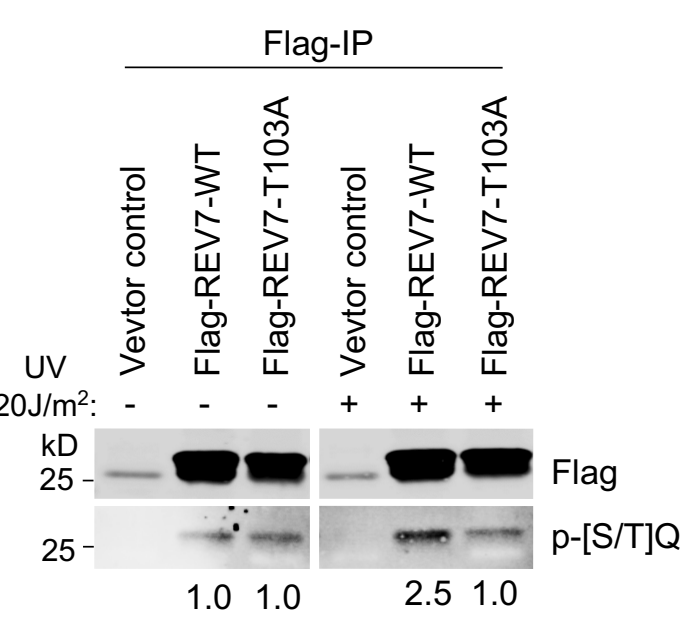

D

Closed

phos-Rev7-T103

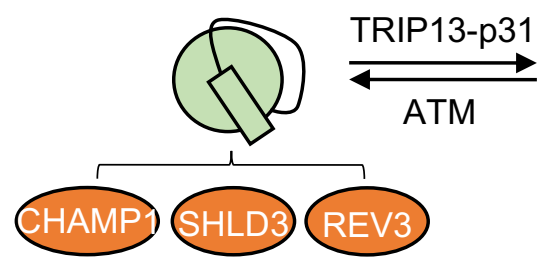

unphos-Rev7-T103



Seatbelt

C

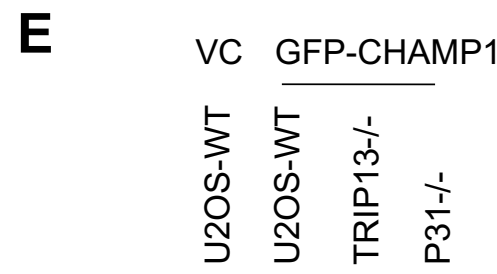

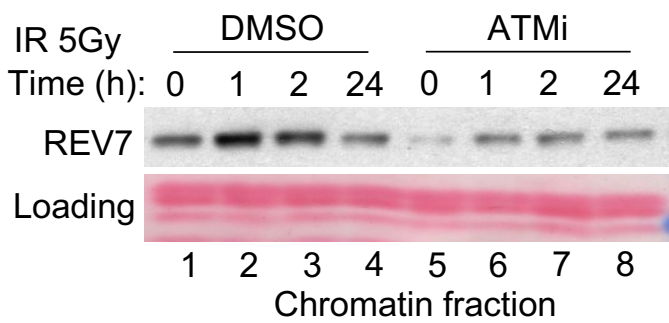

Flag-R7-WT Flag-R7-T103A

Time (h): $\begin{array}{llllllll}0 & 1 & 2 & 4 & 0 & 1 & 2 & 4\end{array}$

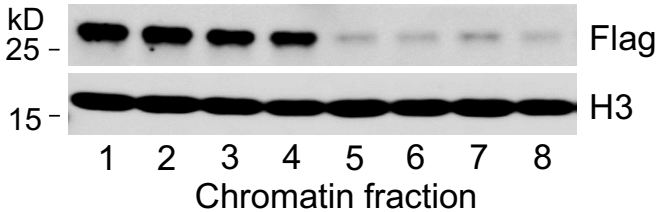

$5 \%$ input

REV7



F



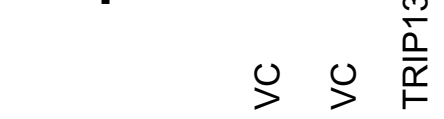

GFP-CHAMP1 - $+\quad+$

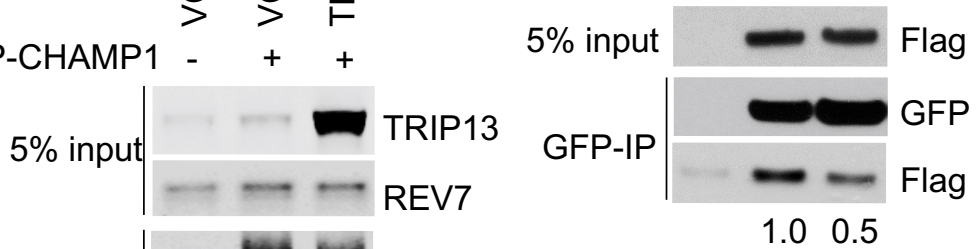

\begin{tabular}{c|c|c|c|c|c|c|c|} 
GFP-CHAMP1 & TRIP13 \\
REP-IP & REV7
\end{tabular}

$\begin{array}{ll}1.0 & 0.2\end{array}$

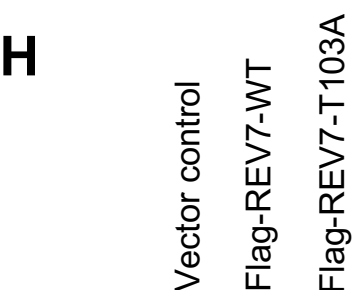

$5 \%$ input

Flag-IP ovid

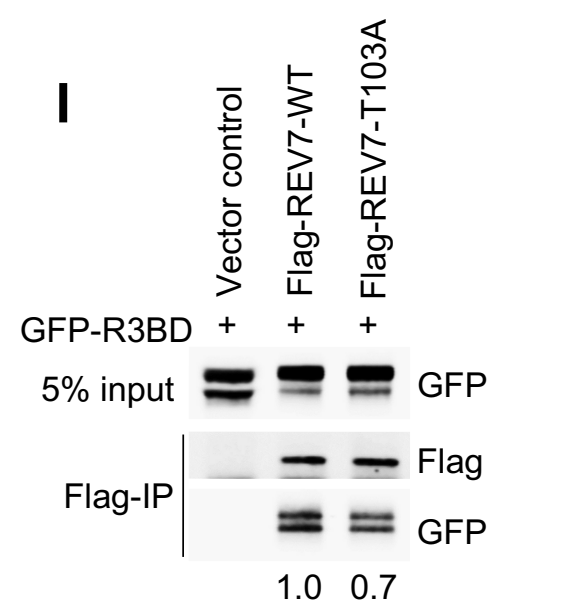

CHAMP1

Flag

CHAMP1 
A

$$
\begin{aligned}
& \text { 虽 } \\
& \text { य } \frac{0}{4} \frac{1}{0}
\end{aligned}
$$

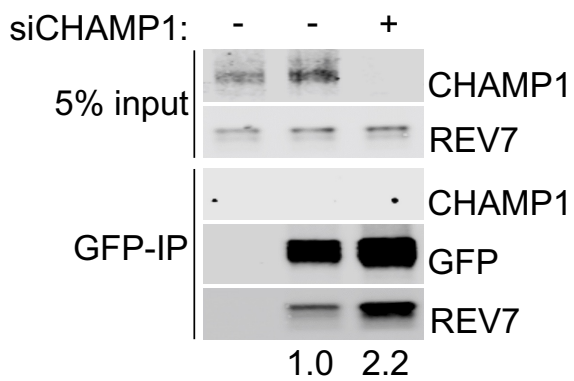

C
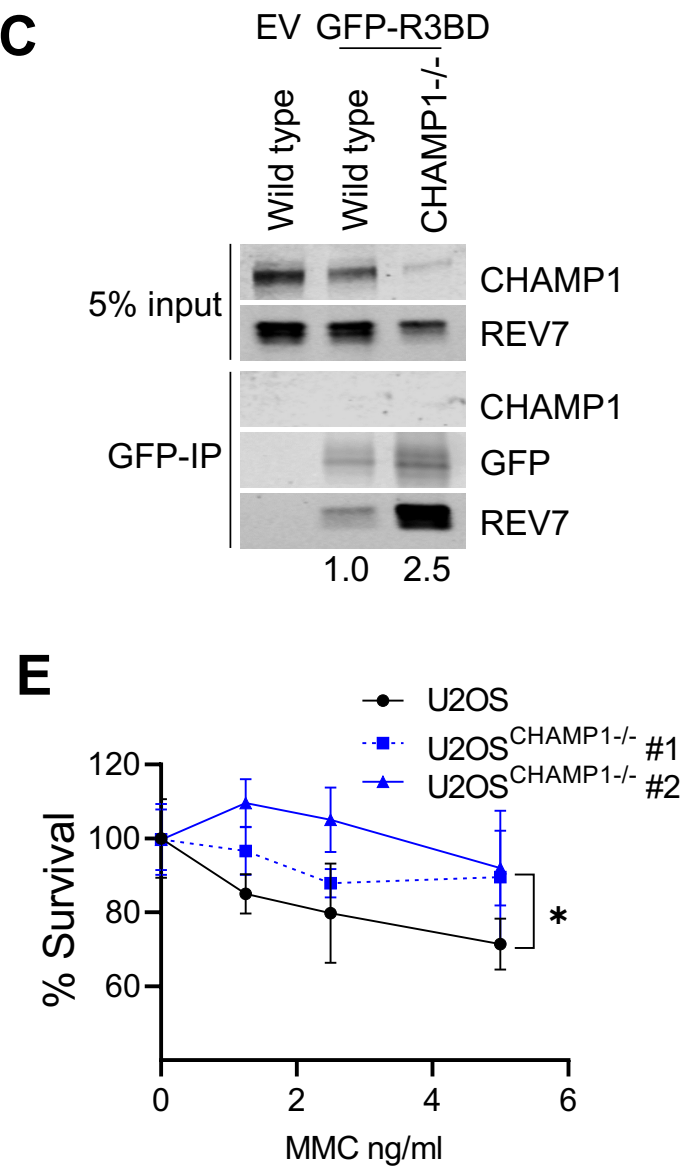

B

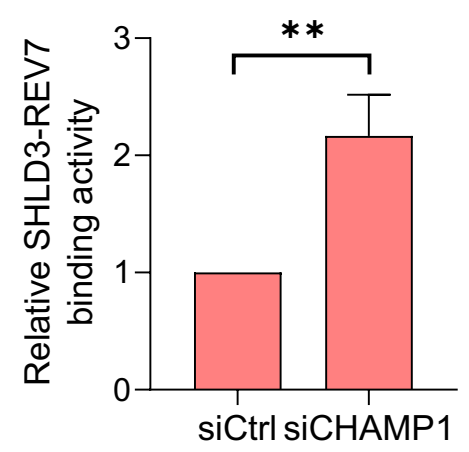

D

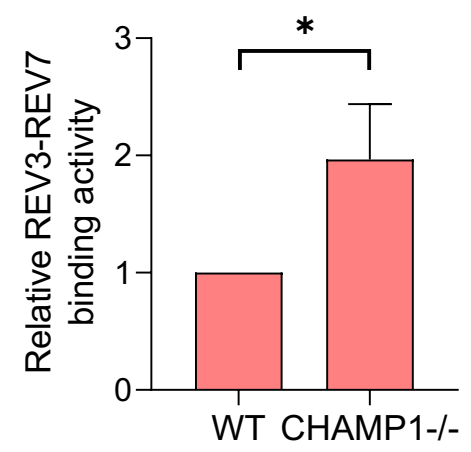

F

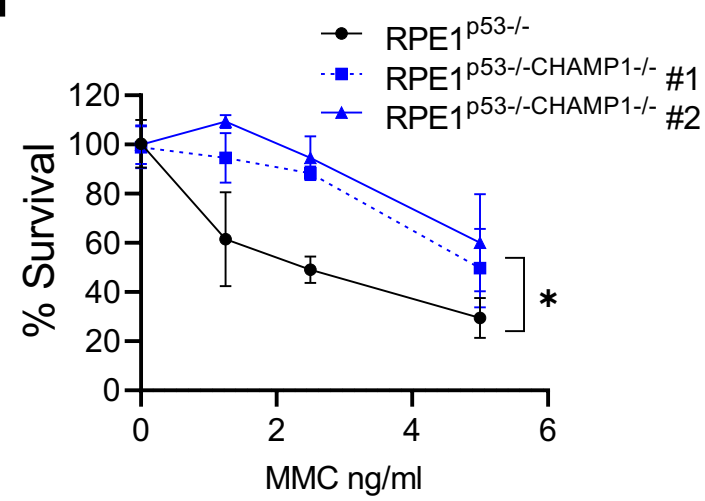


Fig 4. The REV7 binding activity of CHAMP1 is not required for the CHAMP1 chromosome alignment function


RPE1 ${ }^{\text {p53-/-CAMP-/- }}$

RPE $1^{\text {p53-/-CAMP-/- }}$ 
Fig 5. CHAMP1 regulates HR through REV7

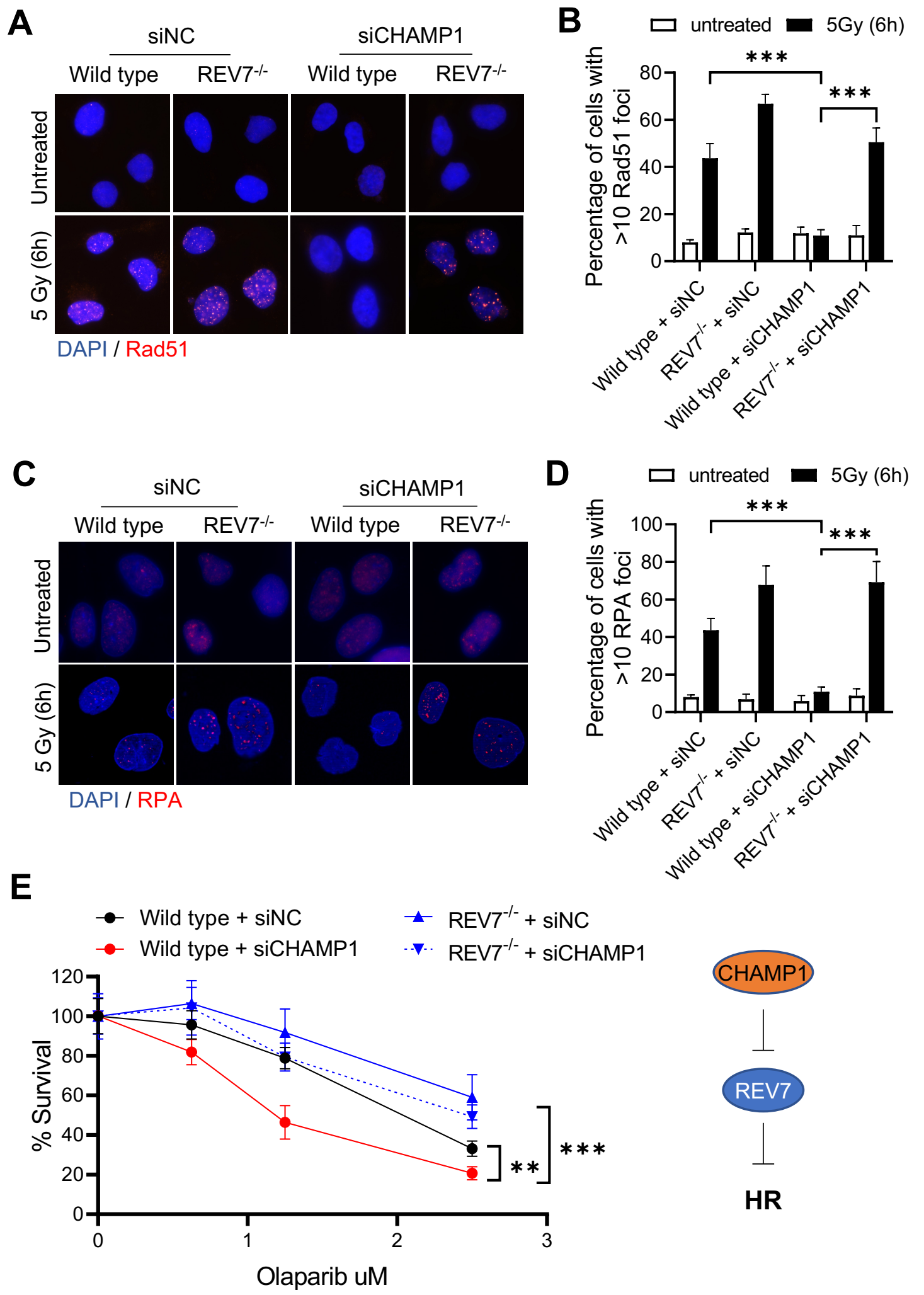




\section{Fig 6. CHAMP1 overexpression is common in tumors with underlying HR deficiency and correlates with poor cancer patient prognosis}

A

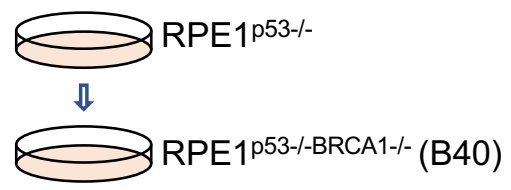

Niraparib/Liparid $\overline{\bar{n}}$
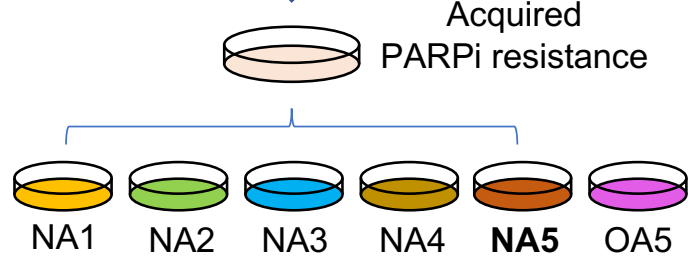

Niraparib

Olaparib

C

TCGA combined cancer types with Wildtype BRCA1 and BRCA2

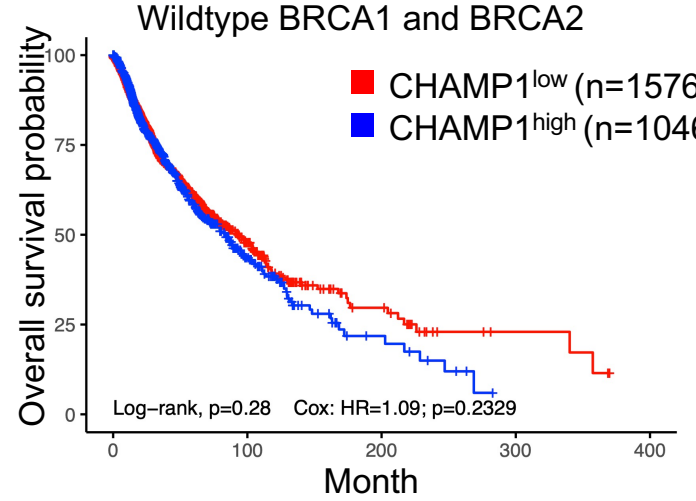

E

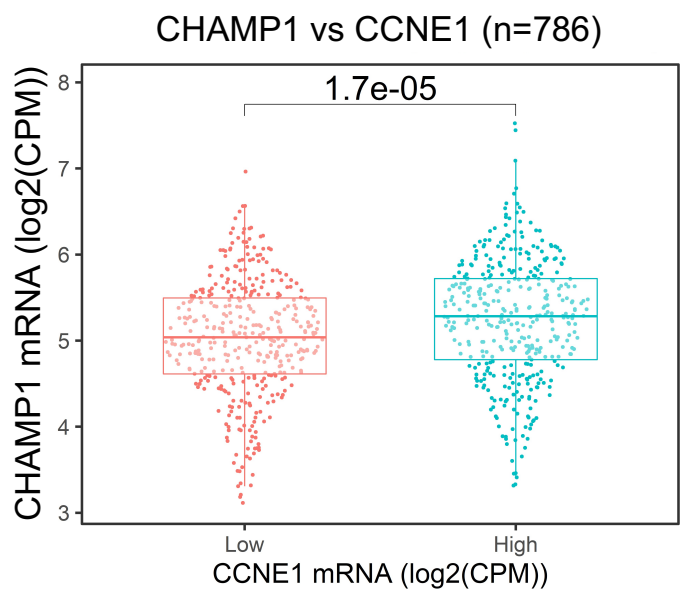

B

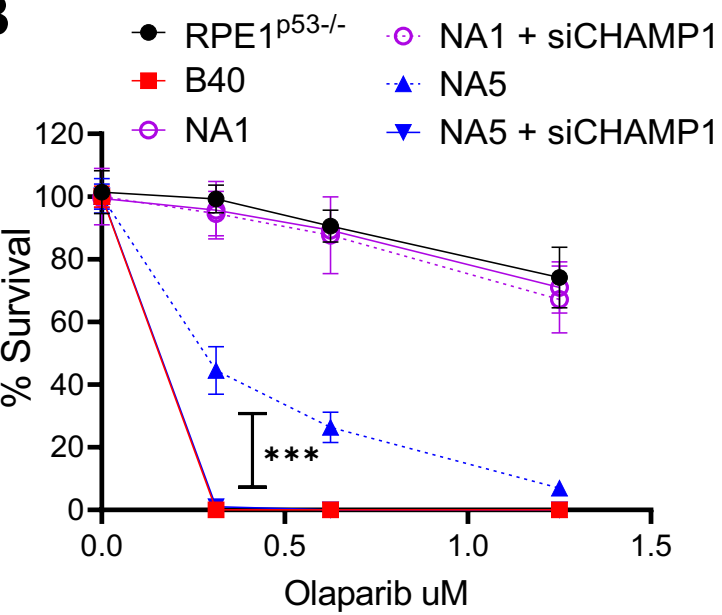

D

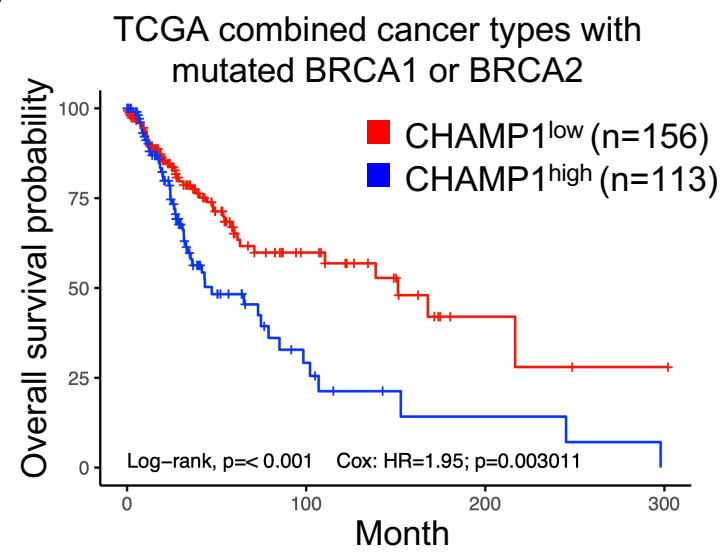

$\mathbf{F}$

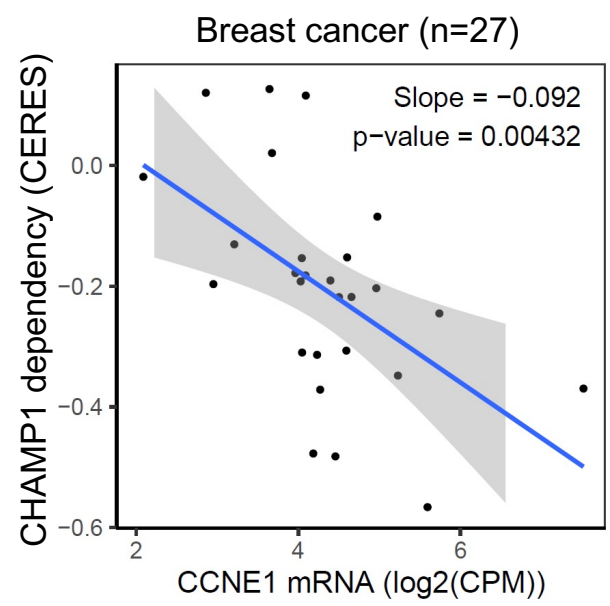


Fig 7. POGZ is epistatic with CHAMP1 in Homologous Recombination
